\title{
Comparison of Cyssor analogues in the cysteine-specific chemical cleavage of ovalbumin
}

\author{
THOMAS J. HOLMES, Jr. and RICHARD G. LAWTON* \\ Department of Medicinal Chemistry, College of Pharmacy, University of Minnesota, \\ Minneapolis, Minnesota, \\ *Department of Chemistry, University of Michigan, Ann Arbor, Michigan, USA
}

Received 18 March, accepted for publication 23 June 1983

\begin{abstract}
A comparison of analogous benzoquinonediimide protein cleavage reagents is presented. While the 2-methoxy derivative reveals no apparent advantage over the previously reported 2-methyl-N $N^{1}$-benzenesulfonyl- $N^{4}$-bromoacetyl-benzoquinonediimide (Cyssor I; Sigma Chemical Company), the 2-chloro analogue reveals significant cleavage of ovalbumin under identical solvent conditions, but without the application of heat. This improvement might allow application of this chemical cleavage technique to particularly temperature-sensitive proteins.
\end{abstract}

Key words: benzoquinonediimides; Cyssor analogues; cystcine modifjcation; protein cleavage

In attempts to improve upon the selective chemical cleavage of proteins at cysteine residues ultilizing the quinonediimide (Cyssor) reagents (1), a number of analogues have been prepared and recently reported (2). Two of these derivatives have been compared to the original 2-methyl- $N^{3}$-benzenesulfonyl- $N^{4}$. bromoacetyl benzoquinonediimide (la) under identical conditions and the observed differences and advantages are reported herein. It is assumed that each of these reagents reacts similarly with ovalbumin by initial nucleophilic addition of free protein sulfhydryl functions to the quinonoid nucleus in a position "para" to the ring substituent; followed by intramolecular bromide displacement to generate an unstable sulfonium ion whose subsequent decomposition leads to cleavage of the cysteinyl$N$-peptide bond (as described with supporting evidence in our original publication (1)). No

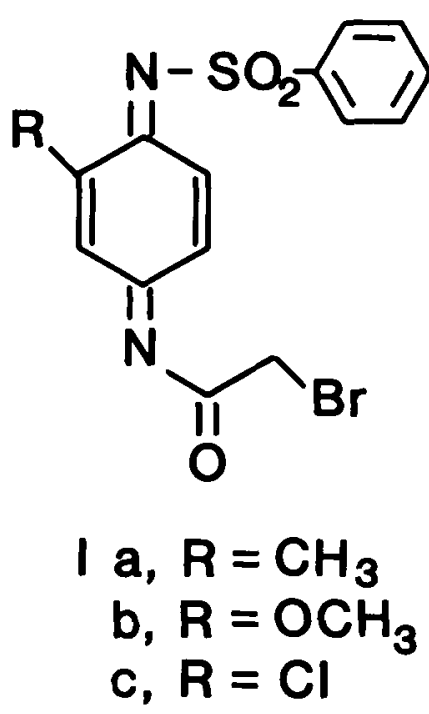

SCHEME 1 
contradictory evidence for either of these derivatives (Meo, $\mathrm{Cl}$ ) has been observed in model studies with $N$-acetyl cysteine as the substrate.

\section{MATERIALS AND METHODS}

The Cyssor analogues $(\mathrm{Ia}-\mathrm{c})$ were prepared in our laboratory by synthetic methods which have been described in detail elsewhere (2). The compound, $2 \cdot$ methyl $-N^{1}$ benzenesulfonyl$N^{4}$-bromoacetylquinonediimide is available from Sigma Chemical Company under the trade name of Cyssor I. Ovalbumin was purchased commercially from Nutritional Biochemicals Corporation. In general, the reactions of ovalbumin and the various quinonediimides were carried out in the following manner. To $100 \mu \mathrm{l}$ protein solution $(2 \mathrm{mg} / \mathrm{ml} ; 4.5 \mathrm{nmol} /$ $100 \mu \mathrm{l}$ in $0.1 \mathrm{~N}$ HOAc with $0.5 \%$ SDS and $0.001 \mathrm{M}$ EDTA) at room temperature was added all at once $10 \mu \mathrm{l}$ quinonediimide dissolved in acetone $(32 \mathrm{mg} / \mathrm{ml} ; 800 \mathrm{nmol} / 10 \mu \mathrm{l})$. All solvents were flushed with nitrogen prior to use. At these specific concentrations a precipitate forms immediately upon mixing, but the solution clears completely within a few minutes. The solutions were sealed in glass ampules under vacuum and allowed to stand for $20 \mathrm{~h}$ at room temperature in the dark. Any color which formed in the solution (usually orange, but dark purple with the 2-chloro-substituted quinonediimide) faded upon standing at room temperature. It was found that at the end of this room temperature incubation period a heating period of $1 \mathrm{~h}$ at $80^{\circ}$ was usually necessary to cause fragmentation of the protein. After heating, the reaction was quenched by adding $10-20 \mu \mathrm{l}$ aqueous $1 \%$ SDS containing 1\% 2mercaptoethanol. This mixture was then heated for at least $1 \mathrm{~min}$ at $100^{\circ}$ to insure complete reduction and denaturation of the components prior to SDS gel electrophoresis. Finally, $25 \mu \mathrm{l}$ of $0.05 \%$ bromophenol blue in $50 \%$ aqueous glycerol was added and the solution $\mathrm{pH}$ was adjusted to above $\mathrm{pH} 4.6$ with a small amount of $28 \%$ ammonium hydroxide solution.

This mixture was then applied directly to $7 \mathrm{~mm} \times 100 \mathrm{~mm}$ polyacrylamide gel $(10 \%$ acrylamide; 1:38 BIS/acrylamide cross-linking) for electrophoretic separation. The method of
SDS gel electrophoresis employed was that of Weber \& Osborn (3). Separation was carried out at a constant current of $4 \mathrm{~mA} / \mathrm{gel}$ for a period of approximately $7 \mathrm{~h}$. The gels were stained in $0.025 \%$ Coomassie Brilliant Blue for $12-18 \mathrm{~h}$ and subsequently destained by diffusion. Densitometer tracings of the gels were performed at $550 \mathrm{~nm}$ in $7 \%$ acetic acid on a Beckmann-DU spectrophotometer equipped with an automatic gel scanner attachment. The gels were stored at room temperature in $7 \%$ aqueous acetic acid in the dark.

\section{RESULTS AND DISCUSSION}

The ready availability of ovalbumin (MW 44000) from commercial sources and the presence of four cysteinyl sulfhydryl functions in its native form made it a reasonable choice as a standard of comparison for the Cyssor cleavage reagents. The complete primary amino acid sequence of ovalbumin has recently been determined (4). Although composition (5) and sulfhydryl reactivity (6) have been reported to vary, consistent use of a single batch of this protein has allowed the qualitative and semi-quantitative evaluation of reaction conditions to aid in the development of this series of reagents. A large number of trials under a variety of reaction conditions have led to the definition of this cleavage procedure for ovalbumin which is described under Materials and Methods (7). Past application of 2-Me-Cyssor to the cleavage of reduced bovine pancreatic ribonuclease supports the expected generality of this method to the cleavage of a variety of cysteine-containing proteins (1). No attempt, thus far, has been made by us to characterize in detail the frag. ments obtained by cleavage of ovalbumin with these reagents $(1 \mathrm{a}-\mathrm{c})$. Also, no attempt to identify or quantitate the colored materials observed upon initial addition of these reagents to the protein has been carried out.

The cleavage results recorded in Figs. 1-3 may be viewed in a semi-quantitative manner. That is, since the quantity of Coomassie blue which is bound to any one of the cleavage fragments is likely to be a function of the molecular weight (and possibly the amino acid composition) of that fragment; lower molecular 


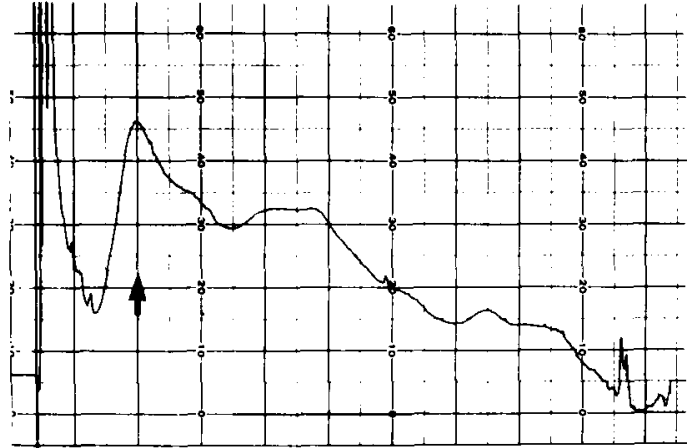

(a)

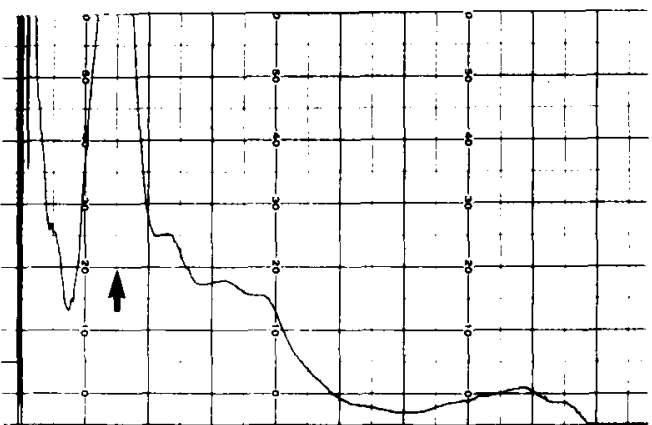

(b)

FIGURE 1

(a) Tube gel densitometer tracing of Coomassie Blue stained protein fragments obtained from treatment of ovalbumin with 2-Me-Cyssor (Ia) as described in the Methods section (heated). (b) Gel densitometer tracing of ovalbumin slight fragmentation obtained when the 2-Me-Cyssor reaction is unheated. All gels were traced left to right, high molecular weight to low molecular weight. The arrow indicates the position of ovalbumin which has been treated under identical conditions without the presence of cleavage agent (full trace previously published in reference 1).

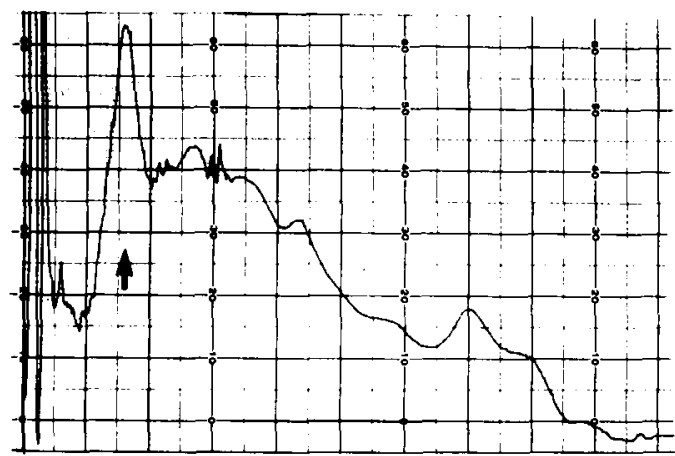

(a)

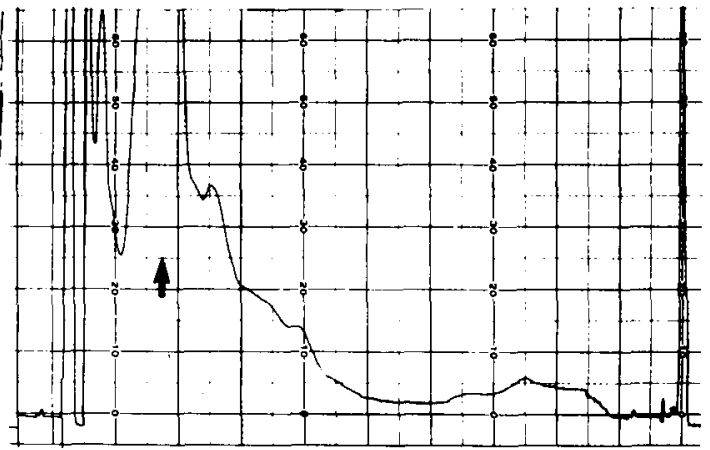

(b)

\section{FIGURE 2}

(a) Gel densitometer tracing of ovalbumin treated with 2-MeO-Cyssor $(\mathrm{Ib})$ and heated. (b) Densitometer tracing of ovalbumin slight fragmentation obtained when the 2-MeO-Cyssor reaction is unheated.

weight fragments appear less dense utilizing this detection technique. However, since binding of Coomassie blue to uncleaved ovalbumin should remain constant (especially since this data represents a single experimental set in which all reaction conditions and gel runs, staining and destaining, were performed identically), its loss or presence should be fairly quantitative with respect to the gel scans presented. In these studies resolution of protein fragments on the gels was sacrificed by using relatively large amounts of protein to detect low level fragmentation and by minimizing sample handling through direct application of crude reaction mixture.

With this in mind, then, the following observations might be made. Considering the data in Figs. 1-3 (sections (a) only) it is observed that 


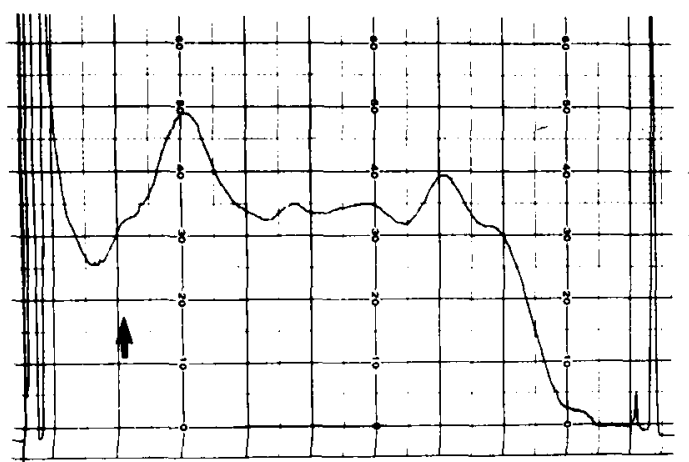

(a)

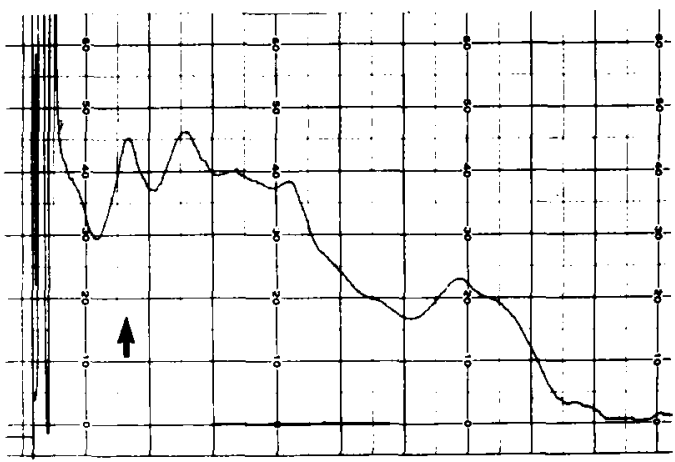

(b)

\section{FIGURE 3}

(a) Gel densitometer tracing of ovalbumin treated with $2-\mathrm{Cl}-\mathrm{Cyssor}$ (Ic) and heated; (b) Densitometer tracing which shows significant cleavage of ovalbumin upon treatment with 2 -Cl-Cyssor without the application of heat.

each of three Cyssor analogues $(I a-c)$ produced a high degree of protein cleavage when the $1 \mathrm{~h}$ heating period $\left(80^{\circ}\right)$ was employed. Based upon the relative amounts of ovalbumin remaining, the order of cleavage efficiency for the heated reactions would be $2-\mathrm{Cl}>2-\mathrm{Me}>2 \cdot \mathrm{MeO}$ for the Cyssor analogues. This relative cleavage efficiency is seen to be identical and even more pronounced in Figs. 1-3 [sections (b)] where no heat was applied after the initial room temperature incubation period. It is observed that (relative to the amount of ovalbumin remaining) there is very little protein cleavage with the $2-\mathrm{Me}$ and $2 \cdot \mathrm{MeO}$ analogues, whereas 2-Cl-Cyssor shows significant fragmentation of ovalbumin without the application of elevated temperatures (although less than that observed in the heated sample, Fig. 3(a)).

These results are consistent with the importance of electronic effects in the proposed cleavage mechanism. Since it has been shown that the addition of mercaptans to quinonediimides bearing any of these single substitutes (Me; $\mathrm{MeO} ; \mathrm{Cl}$ ) leads predominantly to "para" $(2,5)$ substitution (8), these electronic effects might be more important in mechanistic events subsequent to the initial sulfhydryl addition. The relative cleavage efficiency of these Cyssor analogues as indicated by fragmentation of ovalbumin (Figs. 1-3(a) or (b)) under identical conditions appears to correlate with the Ham- mett sigma plus constants $\left(\sigma_{\mathrm{p}}^{+}\right)(9): \mathrm{Cl}(+0.11)$; $\mathrm{Me}(-0.31) ; \mathrm{MeO}(-0.78)$, which quantitate the effects of these substituents upon reactions which involve the development of positive charge (e.g. the proposed sulfonium ion intermediate) in the transition state. However, since the rate-determining step in this cleavage process might be beta-elimination of the reagent-induced sulfonium ion intermediate leading to charge neutralization, correlation with the charge-independent Hammett constant $\left(\sigma_{\mathrm{p}}\right)$ might be more appropriate: $\mathrm{Cl}(+0.23)$; $\mathrm{Me}(-0.17) ; \mathrm{MeO}(-0.27)$. Although either of these correlations may be applicable, it is impossible to distinguish these influences at this time. In either case, the beneficial effect of halogen-substitution is apparent and these observations will serve to direct the development and refinement of this chemical cleavage technique in the future.

\section{ACKNOWLEDGMENTS}

Financial support for this work was provided through NDEA Title IV and NIH Traineeship programs.

\section{REFERENCES}

1. Holmes, Jr., T.J. \& Lawton, R.G. (1977) J. Am. Chem. Soc. 99, 1984-1986

2. Holmes, Jr., T.J. \& Lawton, R.G. (1983) J. Org. Chem. 48 , in press 
T.J. Holmes, Jr. and R.G. Lawton

3. Weber, K. \& Osborn, M. (1969) J. Biol. Chem. 244, 4406-4412

9. Brown, H.C. \& Okamoto, Y. (1958) J. Am. Chem. Soc. 80, 4979-4987

4. Nisbet, A.D., Saundry, R.H., Moir, A.J.G., Fothergill, L.A. \& Fothergill, J.E. (1981) European J. Biochem. 115, 335-345

5. Iwase, H., Kato, Y. \& Hotta, K. (1981) J. Biol. Chem. 256, 5638-5642

Address:

6. Fothergill, L.A. \& Fothergill, J.L. (1970) Biochem. J. 116, 555-561

Thomas J. Holmes, Jr., Ph.D.

Department of Medicinal Chemistry

7. Holmes, Jr., T.J. (1975) Ph.D. Thesis, University of Michigan, University of Microfilms, Ann Arbor, Michigan

College of Pharmacy

8-170 Health Sciences Unit F 308 Harvard Strect S.E.

8. Adams, R. \& Reifschneider, W. (1958) Bull. Soc. Chim. Fr., 23-65

Minneapolis, Minnesota 55455

USA 